\title{
Carbon Nanotube Solar Cells
}

\author{
Colin Klinger, Yogeshwari Patel, Henk W. Ch. Postma*
}

Department of Physics and Astronomy, California State University Northridge, Northridge, California, United States of America

\begin{abstract}
We present proof-of-concept all-carbon solar cells. They are made of a photoactive side of predominantly semiconducting nanotubes for photoconversion and a counter electrode made of a natural mixture of carbon nanotubes or graphite, connected by a liquid electrolyte through a redox reaction. The cells do not require rare source materials such as $\mathrm{In}$ or $\mathrm{Pt}$, nor high-grade semiconductor processing equipment, do not rely on dye for photoconversion and therefore do not bleach, and are easy to fabricate using a spray-paint technique. We observe that cells with a lower concentration of carbon nanotubes on the active semiconducting electrode perform better than cells with a higher concentration of nanotubes. This effect is contrary to the expectation that a larger number of nanotubes would lead to more photoconversion and therefore more power generation. We attribute this to the presence of metallic nanotubes that provide a short for photo-excited electrons, bypassing the load. We demonstrate optimization strategies that improve cell efficiency by orders of magnitude. Once it is possible to make semiconducting-only carbon nanotube films, that may provide the greatest efficiency improvement.
\end{abstract}

Citation: Klinger C, Patel Y, Postma HWCh (2012) Carbon Nanotube Solar Cells. PLoS ONE 7(5): e37806. doi:10.1371/journal.pone.0037806

Editor: Arum Han, Texas A\&M University, United States of America

Received August 2, 2011; Accepted April 24, 2012; Published May 24, 2012

Copyright: (C) 2012 Klinger et al. This is an open-access article distributed under the terms of the Creative Commons Attribution License, which permits unrestricted use, distribution, and reproduction in any medium, provided the original author and source are credited.

Funding: The authors have no support or funding to report.

Competing Interests: The authors have declared that no competing interests exist.

*E-mail: postma@csun.edu

\section{Introduction}

Solar cells have great potential as an alternative energy source because of the enormous amount of available energy and its distributed nature that may enable a distributed power generation grid [1]. However, for solar energy to be cost-effective on a utility scale, the price of purchase, installation, operation and maintenance over the lifetime of a solar panel per kWh generated must compare favorably to current power generation technology, which for fossil-fuel based generation is $0.03-0.05 \$ / \mathrm{kWh}$ [2]. Improvements are being made to solar cells to 1 ) increase the efficiency, and 2) lower the price. For instance, solar concentrators are being developed that focus solar light reflecting off a large mirror on a solar cell with a smaller surface area. Multi-junction devices are being developed that use junctions between materials with different band gaps to capture a greater number of photons and limit loss of excess photon energy when the excited high-energy electron relaxes to the Fermi level.

Gratzel cells [3], also known as Dye-Sensitized Solar Cells (DSSCs), offer a particularly interesting path to cost-effective solar power. By sacrificing some efficiency but offering a greater reduction in cost, the total price per $\mathrm{kWh}$ can be reduced considerably. While this initial argument for DSSGs is very compelling, it is worth noting that the current state-of-the art DSSCs have efficiencies that rival their solid-state counterparts [46]. Another advantage of DSSCs is that they operate well in lowlight and overcast conditions. DSSCs typically consist of a transparent semiconducting film on conducting glass that functions as a photo-active electrode (figure la, top). A glass plate is coated with $\mathrm{Pt}$ and acts as the counter electrode (figure la, bottom). Light-sensitive dye molecules are adsorbed on a semiconducting material on another slide and the assembly is immersed in an electrolyte, typically iodide-triiodide $\left(I^{-} / I_{3}^{-}\right)$. An incoming photon with energy $h v$ excites an electron from the dye into the conduction band of the semiconductor and it migrates to the bottom electrode. The electrolyte reduces the dye, creating triiodide $\left(3 I^{-} \rightarrow I_{3}^{-}+2 e^{-}\right)$. The electrons follow the external circuit through the load to the counter electrode. The triiodide migrates through the electrolyte to the $\mathrm{Pt}$ electrode and gets reduced, thereby completing the circuit. The transparent semiconductor is typically made of nanoporous $\mathrm{TiO}_{2}$. Using a nanoporous material significantly increases the surface area available for dye molecules but at the same time limits the electron migration rate. Different transparent semiconductors are being studied with higher mobility, such as nanowire-based electrodes $[7,8]$. The liquid electrolyte is not very stable at the wide range of temperatures solar cells typically are exposed to, so high-mobility solids are being investigated as well $[9,10]$, culminating recently in a record $12 \%$ conversion efficiency [6]. Various dyes have been used in DSSCs, ranging from metal-free organic dyes [11] through highly efficient Ru-based organic dyes such as 'N3 dye' [12,13] and 'black dye' [14-17] to engineered semiconductor quantum dots with a very high extinction coefficient [18]. $\mathrm{C}_{60}$ has been shown to work as a 'dye' as well [19,20]. Carbon nanotubes (CNTs) [21,22], offer a potentially cheaper and easier alternative to these materials. They are photo active, highly conductive, strong, and chemically inert. Carbon nanotubes can be synthesized in multiple ways such as chemical vapor deposition or laser ablation. The natural ratio of assynthesized carbon nanotubes is $2 / 3$ semiconducting to $1 / 3$ metallic.

Here, we present proof-of-concept solar cells that are entirely made of carbon nanotubes, carbon-nanotube-based solar cells (CNSGs, figure 1b). They are a variation on the DSSC, and potentially offer many advantages beyond DSSCs. 1) No Dye. As these cells use semiconducting CNTs for photo conversion, they 


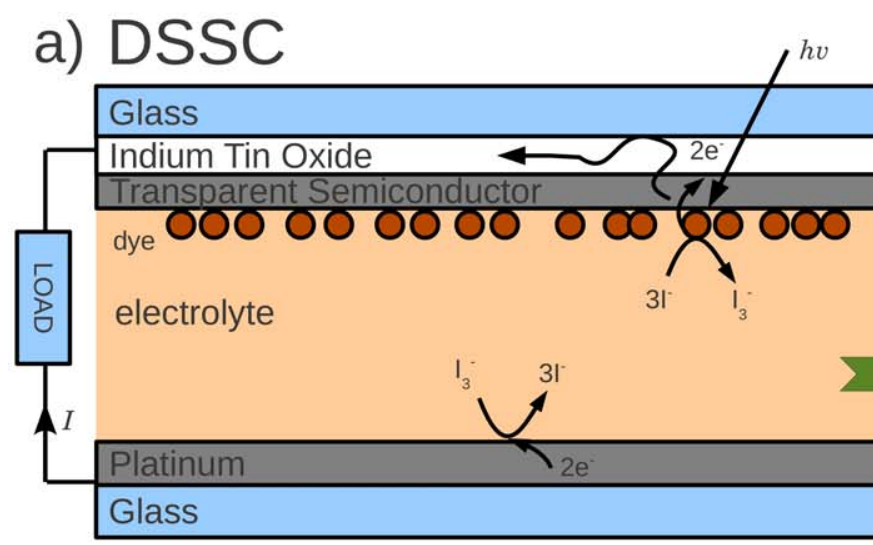

\section{b) CNSC}

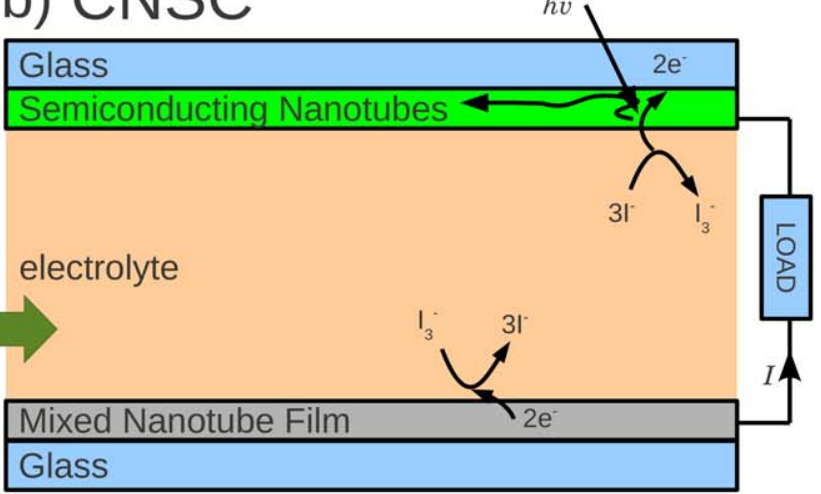

c)

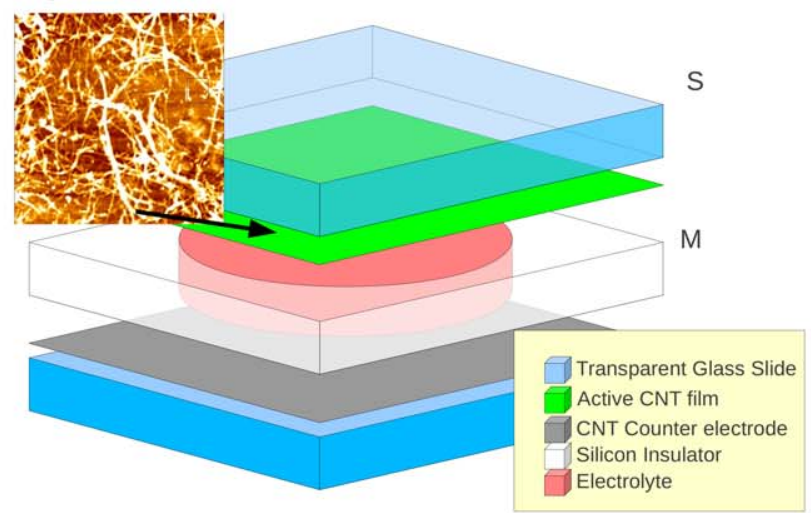

d)

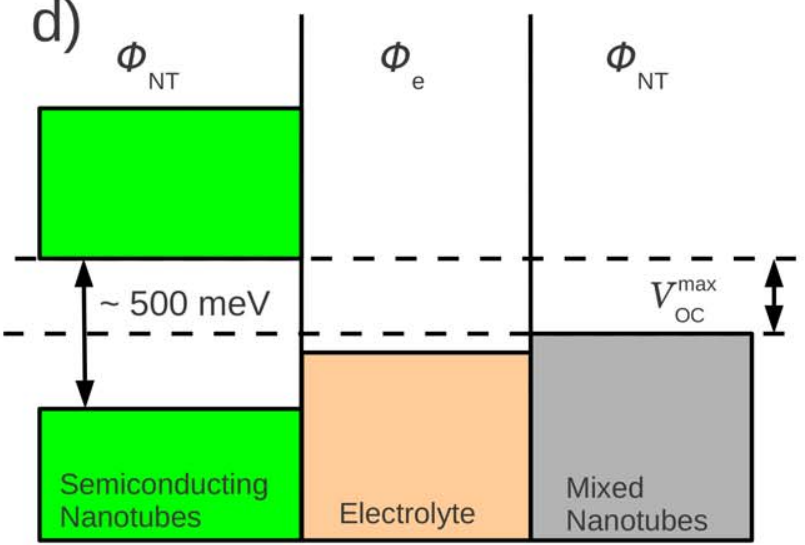

Figure 1. Carbon nanotube solar cells; comparison to Dye-Sensitized Solar Cells (DSSC), construction, and energeticts. a) DSSC. b) Carbon Nanotube Solar Cell, CNSC. c) Layout of a CNSC. The top and bottom glass slides (light blue) are covered in carbon nanotube films which are electrically connected by the iodide-triiodide electrolyte (light red) that is contained by the silicone separator (white). The top film (green) is the photoactive electrode, while the bottom electrode (grey) is the counter electrode. The inset is an Atomic Force Micrograph of the height of a $2 \times 2 \mu \mathrm{m}$ section of a carbon nanotube film. d) Band diagram of the CNSC. doi:10.1371/journal.pone.0037806.g001
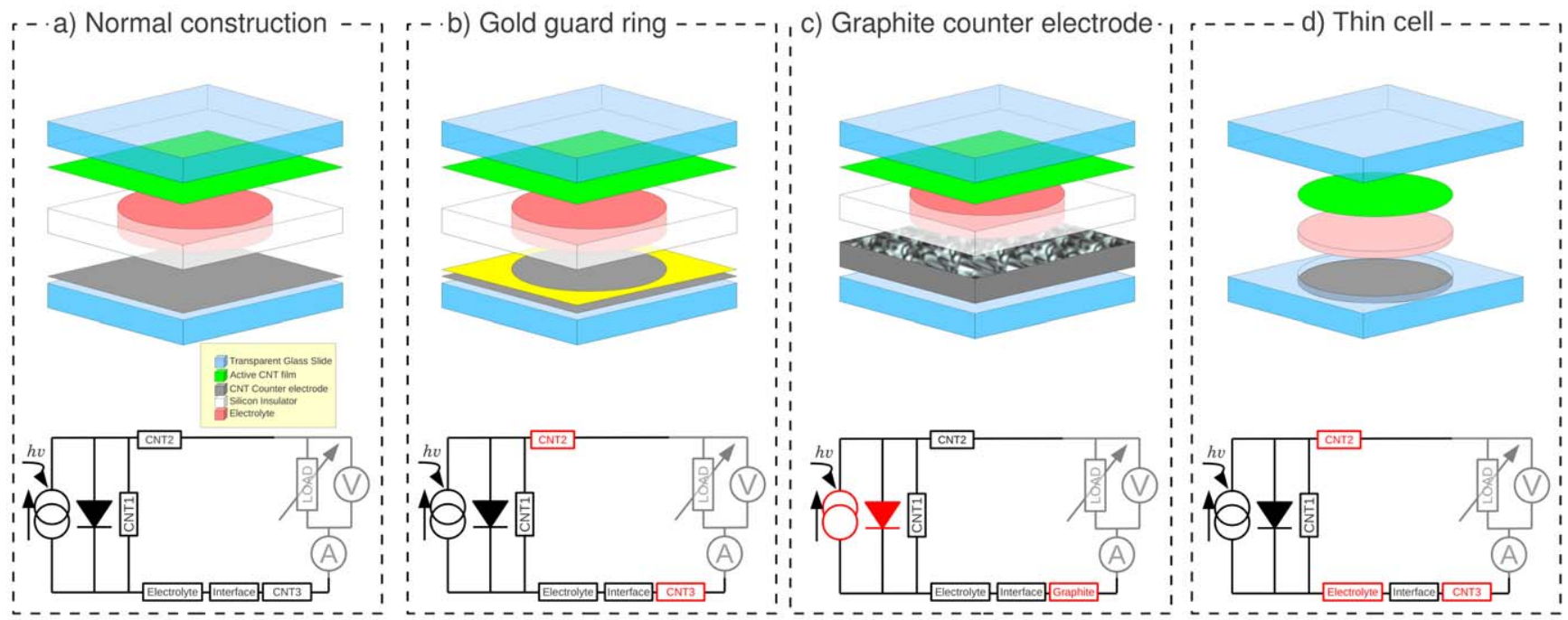

Figure 2. Layout of DSSCs and equivalent circuits. Both basic DSSCs (a) and tested optimization strategies are (b-d) are depicted. The circuit diagram is modeled after [41]. The alternative construction techniques lead to changes in the cell's electrical model, which are highlighted in red. doi:10.1371/journal.pone.0037806.g002 
a)
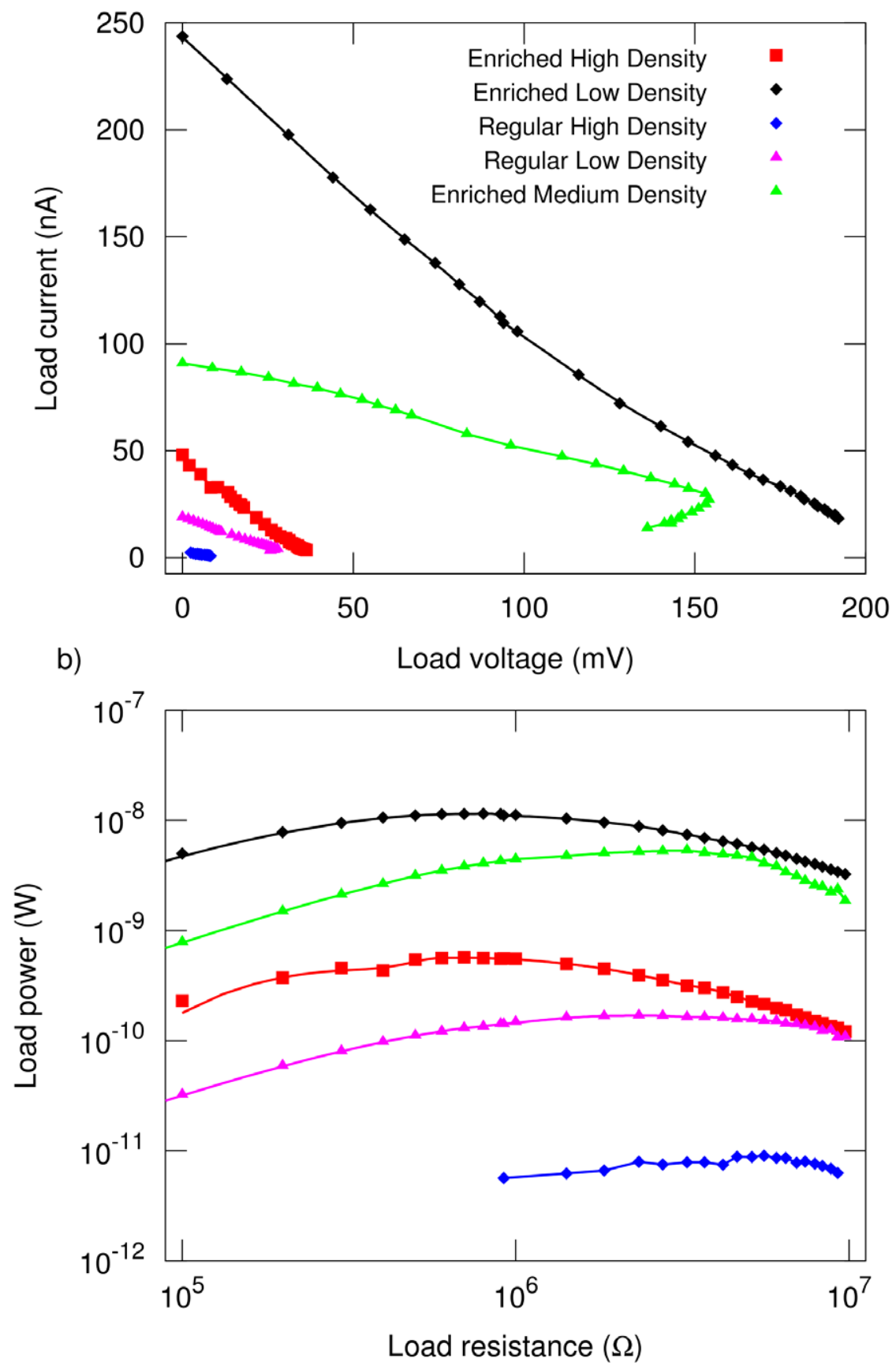

Figure 3. Electrical characteristics of the CNSCs. The extracted parameters are presented in table 2 . a) $I-V$ characteristics of the cells as indicated. b) Power delivered to the load for all cells as described in the legend for a).

doi:10.1371/journal.pone.0037806.g003

do not rely on dyes, which may bleach, severely limiting the useful life of DSSC. 2) No Pt. Pt is often used as counter electrodes and their use in DSSCs represent an undesirable reliance on noble metals which may inhibit the use of DSSCs on a large, i.e. utility, scale. In addition, Pt has been reported to degrade due to the contact with the electrolyte [23]. Carbon nanotubes, in contrast, are chemically inert, and indeed show promising characteristics as counter electrodes [24-27]. 3) No In. As the carbon nanotube film itself is a transparent conductor, the use of a conducting coating made of, e.g. $\mathrm{InSnO}$, is not required, eliminating the need for the exceedingly rare Indium. 4) The application of carbon nanotubes to the glass slides is a low temperature spray-coating process. In addition, these CNSCis multiply the advantages offered by DSSCs over single and multi-junction solar cells that require high-grade semiconductors and clean-room manufacturing. The use of lowgrade materials and resulting projected significant reduction in cost of manufacturing potentially offsets the limited efficiency of these cells when relating the energy produced per dollar spent in manufacturing and installation. 
Table 1. Parameters of CNSCs.

\begin{tabular}{|c|c|c|c|c|c|c|c|c|c|}
\hline & & Cell Type & $R_{\square}$ & $V_{\mathrm{OC}}$ & $I_{\mathrm{SC}}$ & $P_{\max }$ & $R_{\max }$ & $n_{s}$ & $n_{m}$ \\
\hline & & & $\mathrm{k} \Omega / \square$ & $\mathbf{m V}$ & $\mathbf{n A}$ & nW & $\mathrm{M} \Omega$ & a.u. & a.u. \\
\hline - & & Enriched High Density & 3.4 & 43.6 & 47.0 & 0.57 & 0.71 & 521 & 58 \\
\hline - & (black) & Enriched Low Density & 62.7 & 208.5 & 243.4 & 11.50 & 0.82 & 121 & 13 \\
\hline - & (blue) & Regular High Density & 30.2 & 9.3 & 4.4 & 0.01 & 5.55 & 36 & 18 \\
\hline $\boldsymbol{\Delta}$ & (magenta) & Regular Low Density & 50.3 & 21.4 & 19.0 & 0.17 & 2.45 & 29 & 15 \\
\hline $\boldsymbol{\Delta}$ & (green) & Enriched Medium Density & 46.1 & 154.1 & 91.2 & 5.32 & 3.01 & 136 & 15 \\
\hline
\end{tabular}

In addition to CNT-only cells, we report on effiency improvement strategies, using different assembly techniques and using graphite (graphenium) counter electrodes. Graphite has no band gap, is extremely pliable, robust, and provides the ability to shrink the distance between it and the active semiconducting electrode. The cost, relative abundance, ease of introduction into the cell, and lack of need for spray deposition render graphite an attractive counter electrode material.

\section{Results}

We present experimental demonstration of power generation obtained under ambient conditions at solar noon (see Methods section for details) of two types of cells. 1) CNT-only cells: cells are built with identical geometry but different CNT film compositions and thickness. This highlights how film composition affects cell performance (figure 2a). 2) Optimized cells: cells are built with the same CNT film thickness and composition, but with differences in construction techniques to isolate its role in cell efficiency (figure $2 \mathrm{~b}-\mathrm{d}$ ).

\section{CNT-Only Cells (figure 1a)}

The photocurrent $I$ decreases linearly with increasing cell potential applied to the load $V$ (figure $3 \mathrm{a}$ ). We extract the opencircuit voltage $V_{\mathrm{OC}}$ by extrapolating the $I-V$ characteristic to $I=0$ and the short-circuit current $I_{\mathrm{SC}}$ by extrapolating to $V=0$ (table 1). Both $I_{\mathrm{SC}}$ and $V_{\mathrm{OC}}$ of the enriched mixture cells increase with decreasing CNT coverage of the semiconducting active electrode. Similarly, the high-density cell of the regular mixture of nanotubes has a lower $I_{\mathrm{SC}}$ and $V_{\mathrm{OC}}$ than the low-density cell. The power transfer curves (figure $3 \mathrm{~b}$ ) show a peak power transfer of $P_{\max }$ that occurs when the impedance of the load reaches $R_{\max }$. The low-density enriched as well as the low-density regular cells deliver more power to the load than their high-density counterparts. This is consistent with both $I_{\mathrm{SC}}$ and $V_{\mathrm{OC}}$ being larger.

\section{Optimized cells (figure $2 b-d$ )}

We have studied cells with different construction techniques, using CNT electrodes from the same batch. Similar techniques were employed for data analysis as above (figure 4, table 2). The power transfer curves (figure $4 \mathrm{~b}$ ) show a peak power transfer $P_{\max }$ at $R=R_{\max }$. Gold Guard Ring. The presence of the gold guard ring increases $I_{\mathrm{SC}}$ by a factor $\sim 2.5$, while $V_{\mathrm{OC}}$ remains approximately constant. $R_{\max }$ is lower by $\sim 3.5$ and $P_{\max }$ is $\sim 2$ times greater. Graphite Counter Electrode. Both $V_{\mathrm{OC}}$ and $I_{\mathrm{SC}}$ are greater than the normally constructed cell and $P_{\max }$ is $\sim 12$ times greater. Thin Cell. When the enriched side is facing the incident solar radiation ("up"), the power is slightly larger than when the regular side is facing the incident radiation ("down"). Both $V_{\mathrm{OC}}$ and $I_{\mathrm{SC}}$ are lower by factors of $\sim 5$ and $\sim 3$, which in itself is undesirable. However, optimum power transfer occurs at a much lower resistance.

\section{Discussion}

\section{Photocurrent generation and cell voltage}

The linear $I-V$ characteristic phenomenologically indicates the source is purely resistive, and maximum power occurs when the load and source impedance are equal. A figure of merit for solar cells that describes how close its $I-V$ characteristic is to the ideal shape is the fill factor $F F$ which is defined as the ratio of $P_{\max }$ to the maximum power available with the corresponding ideal cell, $F F \equiv P_{\max } /\left(V_{\mathrm{OC}} I_{\mathrm{SC}}\right)$. It ranges from 0 to 1 , where 1 indicates an ideal cell. Ideal cells can supply a constant voltage independent on the load resistance up to the maximum current, when the voltage drops quickly to 0 . Deviations from the ideal fill factor of 1 are usually due to parasitic resistances, such as shunt and series resistances. Shunt resistances affect behavior in the $I-V$ characteristic close to $I_{\mathrm{SC}}$, while series resistances affect performance close to $V_{\mathrm{OC}}$. For our cell, $F F \approx 0.25$. We argue that nanotube resistances 1-3 (figure 2) are responsible for this. Effectively, it means that the diode in the circuit diagram can be neglected. To estimate the number of nanotubes, we use the measured sheet resistance presented in table 1. Our CNT films are in the percolation limit $[28,29]$. We can therefore use the scaling of sheet resistance with number of CNTs to extract the deposited volume of nanotube dispersion $V$, via

$$
R_{\square} \propto\left(V-V_{C}\right)^{-1.5},
$$

where $V_{C}$ is the critical volume that determines the onset of conduction [28]. The volume can then be used to extract the surface density of metallic $n_{m}$ and semiconducting nanotubes $n_{s}$. We assume that the metallic nanotubes dominate the sheet conductance, since their conductance $G_{m}$ is much greater than semiconducting nanotubes $G_{s}$. This assumption holds provided the conductance ratio $G_{m} / G_{s}$ of metallic to semiconducting nanotubes exceeds the semiconducting to metallic abundance ratio $n_{s} / n_{m}$. Single-molecule conductance studies of nanotubes indicate a conductance ratio of $G_{m} / G_{s} \approx 20,[30,31]$ which supports our assumption that metallic nanotubes dominate the sheet conductance. We anticipate that for more enriched semiconducting films than studied here, a more detailed analysis will be required that takes into account the nanotube-nanotube contact resistance as well $[32,33]$. The current-generating capacity of our cells is proportional to the number of semiconducting nanotubes $n_{s}$. Combining both, the open-circuit condition corresponds to an ideal current source $\left(I \propto n_{s}\right)$ connected to CNT1 $\left(R_{\mathrm{CNT1}} \propto R_{\square}\right)$ 
a)

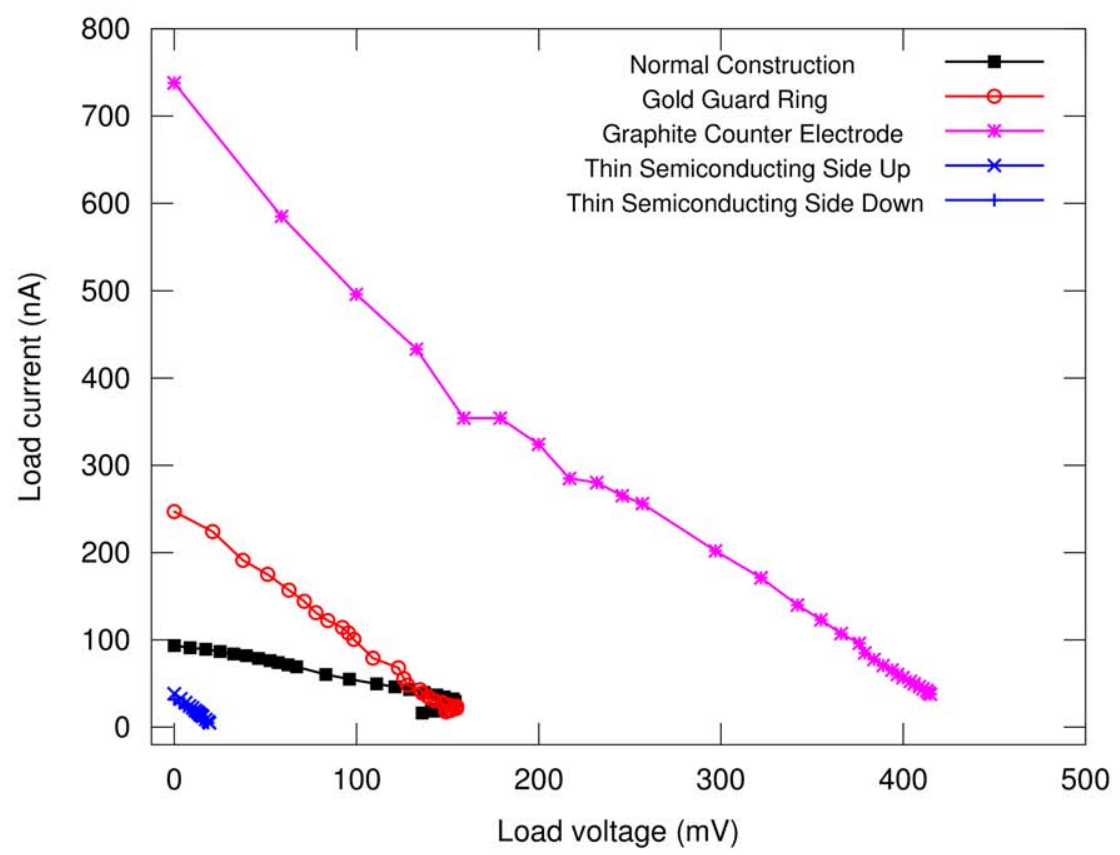

b)

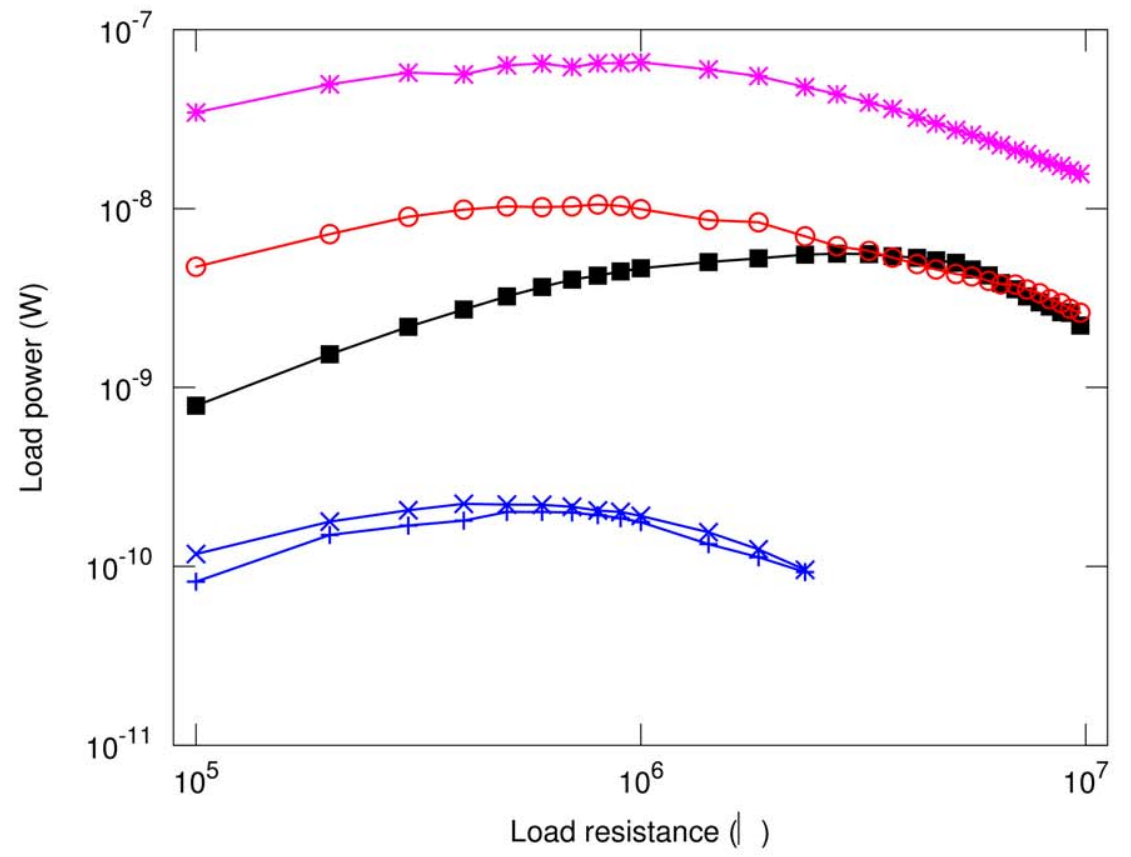

Figure 4. Optimization strategies for CNSCs. The extracted parameters are presented in table 1. a) $I-V$ characteristics of the cells as indicated. b) Power delivered to the load.

doi:10.1371/journal.pone.0037806.g004

and the voltage developed across it will be

$$
V_{\mathrm{OC}} \propto n_{s} R_{\square}
$$

and our data indeed approximately follows this scaling behavior (figure 5a). The outliers at low $V_{\mathrm{OC}}$ are CNT cells where both photoactive and counter electrode are coated with the same composition of carbon nanotubes. Both sides of the cell therefore create a photocurrent in opposite directions, but the light attenuation in the electrolyte breaks this symmetry and causes a directed current, albeit a smaller one and with a smaller voltage. The enriched cells further tilts the balance in favor of the photoactive side, leading to a $V_{\mathrm{OC}}$ that is closer to that expected from the amount of nanotube material deposited on the active side alone.

Our cells have rather large output impedances and cannot maintain constant voltage over a larger range of load impedance. The cell output resistance can be reduced considerably by 
Table 2. Optimized CNSCs.

\begin{tabular}{|c|c|c|c|c|c|}
\hline & \multirow[t]{2}{*}{ Cell Type } & \multirow{2}{*}{$\frac{V_{\mathrm{OC}}}{\mathbf{m} \mathbf{V}}$} & \multirow{2}{*}{$\frac{I_{\mathrm{SC}}}{\mathbf{n A}}$} & \multirow{2}{*}{$\frac{P_{\max }}{\mathbf{n W}}$} & \multirow{2}{*}{$\frac{R_{\max }}{\mathrm{M} \Omega}$} \\
\hline & & & & & \\
\hline [ & Normal Construction & 102.7 & 93.7 & 5.59 & 2.85 \\
\hline - & Gold Guard Ring & 129.5 & 249.5 & 10.46 & 0.81 \\
\hline$*$ & $\begin{array}{l}\text { Graphite Counter } \\
\text { Electrode }\end{array}$ & 438.1 & 733.2 & 65.48 & 0.94 \\
\hline$\times$ & $\begin{array}{l}\text { Thin Semiconducting } \\
\text { Side Up }\end{array}$ & 22.0 & 38.3 & 0.22 & 0.47 \\
\hline+ & $\begin{array}{l}\text { Thin Semiconducting } \\
\text { Side Down }\end{array}$ & 20.9 & 32.0 & 0.20 & 0.58 \\
\hline
\end{tabular}

changing the aspect ratio of the cell, or connecting many cells in parallel. The output voltage can be held constant by a voltageregulation circuit. However, there are many applications that do not require a low output impedance and would therefore work well with CNSCs, e.g. driving an LCD display or an E-Ink screen.

\section{Maximum Open Circuit Voltage}

The band gap of semiconducting carbon nanotubes is related to the nanotube's diameter $d$ through

$$
E_{g}=2 \gamma_{0} \frac{a_{C C}}{d}
$$

where $\gamma_{0}=2.45 \mathrm{eV}$ is the nearest-neighbour overlap integral and $a_{C C}$ is the carbon-carbon distance [30,34-36]. Note that the band gap of semiconducting nanotubes does not depend on the chiral angle. The band gap for a $1.5 \mathrm{~nm}$ nanotube, the average diameter of our nanotube material, therefore amounts to $\sim 500 \mathrm{meV}$. The band diagram is drawn in figure 1d. The 'work function' of the electrolyte is $\Phi_{e}=4.85 \mathrm{eV}$ [37], while the work function for carbon nanotubes is $\Phi_{N T}=4.5 \mathrm{eV}$ [36]. We therefore expect the maximum attainable open circuit voltage $V_{\mathrm{OC}}^{\max } \sim 200-290 \mathrm{mV}$, where the range indicates variations due to the diameter. We observe a maximum voltage of $\sim 200 \mathrm{mV}$. This is expected as nanotubes with a slightly smaller band gap will 'short out' the effect of nanotubes with a slightly larger bandgap.

\section{Solar Power Generation}

The maximum power delivered to the load is a function of $V_{\mathrm{OC}}$ as well as the other resistances in the cell. The composite resistance of the cell is measured by determining at what value $R_{\max }$ of $R_{L}$ maximum power transfer occurs. Since we can model our cells as a voltage source with source voltage $V_{\mathrm{OC}} \propto n_{s} R_{\square}$, the maximum power available is expected to be $P_{\max } \propto V_{\mathrm{OC}}^{2} / R_{\max }$, or

$$
P_{\max } \propto \frac{\left(n_{s} R_{\square}\right)^{2}}{R_{\max }},
$$

and indeed the maximum power appears to follow this behavior approximately (figure 5b).

In summary, both $P_{\max }$ and $V_{\mathrm{OC}}$ behave according to our model that describes the role of film's resistive properties on cell performance.

\section{Optimized Cell Designs}

The gold guard ring causes 1) an increase in $\left.I_{\mathrm{SC}}, 2\right)$ an increase in $\left.P_{\max }, 3\right)$ a decrease in $R_{\max }$, and 4) hardly any change in $V_{\mathrm{OC}}$. We believe this is due to a reduction of the resistance of the nanotube film in contact with the silicone insulator (CNT2 and CNT3, figure 2). The pressure of the insulator on the nanotube film as well as residual shear force during assembly may cause a perturbation of the nanotube film. In addition, the gold lowers the resistance of that part of the nanotube film. Both effects combined act to lower $R_{\mathrm{CNT} 2}+R_{\mathrm{CNT} 3}$. As the open-circuit voltage is independent of $R_{\mathrm{CNT} 2}+R_{\mathrm{CNT} 3}$, the open-circuit voltage should be unaffected by this improvement in design, and indeed we observe $V_{\mathrm{OC}}$ (Normal) $\sim V_{\mathrm{OC}}$ (Gold Guard Ring). The reduction of $R_{\max }$ is also explained by the reduction of $R_{\mathrm{CNT} 2}+R_{\mathrm{CNT} 3}$, and that, in turn, explains the increase of both $I_{\mathrm{SC}}$ and $P_{\max }$.

The employment of a graphite counter electrode instead of a carbonnanotube counter electrode not only improves $I_{\mathrm{SC}}$, but also $V_{\mathrm{OC}}$. The increase in $V_{\mathrm{OC}}$ is due to the use of graphite instead of carbon nanotubes. The increase in $I_{\mathrm{SC}}$ is due to the lower sheet resistance of the graphite as compared to a carbon-nanotube film. The magnitude of the improvement is similar to that accomplished with the gold guard ring improvement, as the maximum power transfer occurs at approximately the same load resistance $\left[R_{\max }\right.$ (Gold Guard Ring) $\sim R_{\max }$ (Graphite Counter Electrode)]. Graphite is preferred over gold, naturally, to reduce cell cost. Both effects combine to increase the power output of the cell by a factor of $\sim 12$.

The reduction of the distance between active and counter electrode for the thin cells can reasonably be expected to lower the resistance of the electrolyte. In addition, as these cells were constructed without a silicone separator, we observe that the maximum power transfer occurs at a much lower resistance. This is due to the absence of the disruptive effect of the silicone separator, which role was elucidated by the study of the gold guard ring device above. The reduction of the electrolyte chamber thickness also has an adverse effect. The electrolyte absorbs less solar radiation than with thicker devices. Therefore, both the enriched (photo active) side, as well as the regular mixture side create a photo current. However, both sources have opposite polarities, causing the effective open-circuit voltage to be reduced as we indeed observe.

\section{Efficiency}

The average solar flux during testing was $770 \mathrm{~W} / \mathrm{m}^{2}$, and the greatest solar power generation was attained with the graphite counter electrode and enriched medium-density CNT active electrode. The efficiency of that cell was $1.8 \times 10^{-5}$. Compared to the all-CNT construction, an improvement of more than a factor 10 was attained. If a cell were constructed with the graphite counter electrode and the low-concentration GNT enriched active electrode, an increase of power by a factor 2 is anticipated. This can be deduced by comparison of the medium density enriched cell to the low density enriched cells with the regular construction. As the graphite counter electrode lowered the output resistance by a factor $\sim 3$, the power output may be larger by a factor 3 as well. Further improvements may be obtained by changing the aspect ratio of the solar cell. In the design reported here, we used effectively square films. Changing the cell design by making the cells wider, will lower the resistance further. An aspect ratio of 10 can then reduce the film resistance by a factor of 10 , causing a reduction of $R_{\max }$, which will improve $P_{\max }$. Our thin cell results indicate that the largest resistance is due to the nanotube film, we therefore believe the efficiency increase with this improvement may be as large as 10-fold. We believe the greatest efficiency increase may be obtained by using CNT source material with a greater fraction of semiconducting nanotubes. The films we used had 90\% semiconducting nanotubes and 10\% metallic nanotubes. 
a)

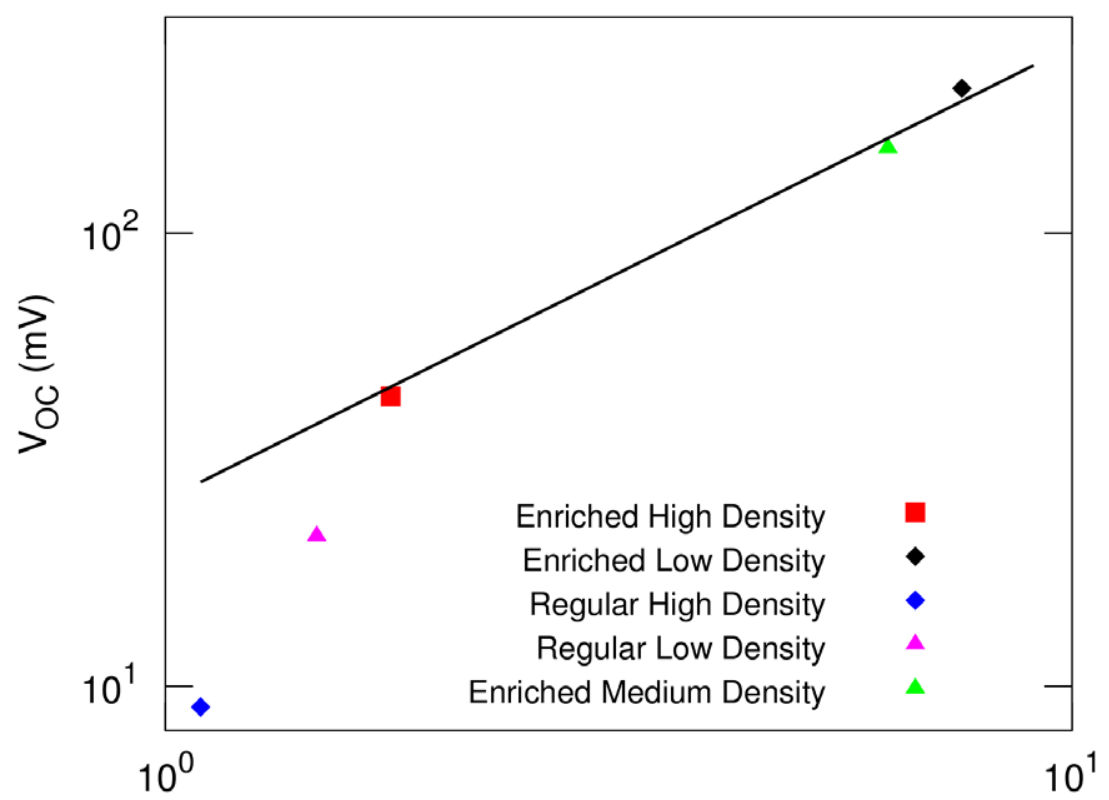

b)

$n_{s} R_{s}$ (a.u.)

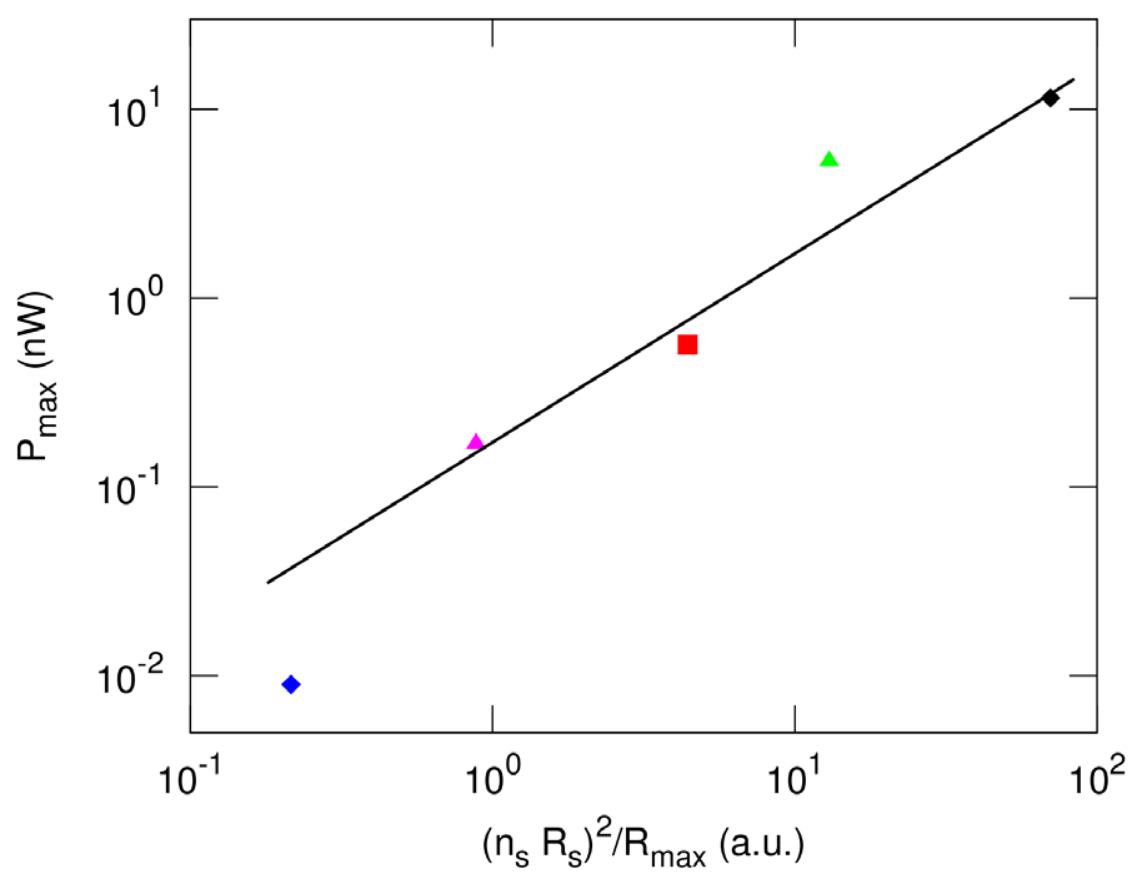

Figure 5. Scaling analysis of CNSC performance. The CNSCs characteristics are determined by the metallic and semiconducting carbon nanotube densities, with symbols corresponding to the cells as in the legend for figure 2. a) $V_{\mathrm{OC}} \propto n_{s} R_{\square}$. b) $P_{\max } \propto\left(n_{s} R_{\square}\right)^{2} / R_{\max }$. doi:10.1371/journal.pone.0037806.g005

As we argued above, the semiconducting nanotubes provide the photo-generated current, but the metallic nanotubes short the load. If one were to use $99 \%$ semiconducting films, the amount of nanotubes could be increased by a factor 10, while still maintaining the same number of metallic nanotubes. As metallic nanotubes are more conductive than semiconducting nanotubes, we assume that the number of semiconducting nanotubes can be increased by this factor 10 without affecting $R_{\square}$ and $R_{\max }$. Future generation cells can then reasonably be expected to deliver 100 times more power, due to the increase of $n_{s}$ by a factor 10 (equation 4). However, at a certain abundance factor of semiconducting to metallic nanotubes, this argument will not hold any longer. Combining all of these improvements may lead to an efficiency of $0.8-5 \%$, where the lower bound is a conservative estimate that every improvement will only contribute half we argued above. We hope the studies reported here will motivate further development of methods to create highly-enriched 
semiconducting CNT source material cost effectively at a large scale.

\section{Materials and Methods}

\section{Cell Construction}

The enriched CNSCs (figure 1) consist of a transparent glass slide covered with an enriched mixture of $90 \%$ semiconducting and $10 \%$ metallic nanotubes (IsoNanotubes-S 90\% Powder, Nano Integris Inc.). These nanotubes have a diameter of $1.2-1.7 \mathrm{~nm}$ and a length of $0.1-4 \mu \mathrm{m}$. Below this is a silicone insulator with a hole filled with electrolyte (iodide-triiodide, Solaronix). The electrolyte is in contact with both carbon nanotube films and acts to reduce the photo-active side as well as close the electrical circuit at the counter electrode. At the bottom is a glass slide covered with a regular mixture of $2 / 3$ semiconducting and $1 / 3$ metallic nanotubes that acts as a counter electrode (Unidym, lot PO-325, formerly Carbon Nanotechnologies Inc.). These nanotubes have a diameter of $0.8-1.2 \mathrm{~nm}$ and a length of $100-1000 \mathrm{~nm}$. Dispersion of carbon nanotubes were made by ultrasonic agitation in 1,2dichloroethane for $1 \mathrm{~h}$ for the regular mixture and $4 \mathrm{~h}$ for the enriched mixture. The dispersion was spray painted with an air brush onto glass substrates in a vented cylindrical enclosure. The slides were rotated while spraying to obtain uniform coverage. Subsequently, the glass slides were heated on a hot plate to evaporate any residual solvent. The resulting film is similar to the well-known bucky paper and it has metallic properties [38-40]. The final solar cell has an exposed surface area of $\sim 4.8 \mathrm{~mm}^{2}$ with a distance of $\sim 2.5 \mathrm{~mm}$ between the electrodes. The glass slides are $1 \mathrm{~mm}$ thick and did not have a conducting coating prior to carbon nanotube application.

\section{Gold guard ring (figure $2 b$ )}

A mask the size of the opening containing the electrolyte was placed onto the glass slide after CNT deposition, followed by $\mathrm{Au}$ deposition. This procedure prevents degration of the metal due to the electrolyte contact.

\section{Graphite cell (figure 2c)}

A cell using graphite (graphenium) as the counter electrode was created. The graphite cell counter electrode construction consists of the same steps for deposition of semiconducting CNTs. A PDMS (Slygard 184 Silicone Elastomer, Dow Corning Corp.) plastic mold with a circular depression was created to house pieces of graphite of different heights. A wire is placed through the PDMS at the height of the bottom of the depression. After graphite deposition the PDMS was filled with electrolyte and the active semiconducting electrode was placed on top of the cell.

\section{Thin Cell (figure 2d)}

A cell with a separation of about $\sim 0.65 \mathrm{~mm}$ between the active $90 \%$ semiconducting electrode and a regular CNT counter electrode was created. A piece of $1 \mathrm{~mm}$ thick glass was locally machined to create a central depression with a connection to a ramped section. The glass was cleaned and masked in the non-

\section{References}

1. Lewis NS (2007) Toward Cost-Effective solar energy use. Science 315: 798-801.

2. Goldemberg J, Johansson TB, Anderson D (2004) World energy assessment: overview 2004 update. United Nations.

3. Gratzel M (2004) Conversion of sunlight to electric power by nanocrystalline dye-sensitized solar cells. Journal of Photochemistry and Photobiology A 164: 3-14.

4. Gao F, Wang Y, Zhang J, Shi D, Wang M, et al. (2008) A new heteroleptic ruthenium sensitizer enhances the absorptivity of mesoporous titania _lm for a machines areas. The glass was sprayed with the $2 / 3$ semiconducting and $1 / 3$ metallic nanotubes mixture. A second piece of glass was masked with the same pattern as the machined glass piece and sprayed with $90 \%$ semiconducting CNTs. The two nanotube electrodes were connected to external electrodes and the cell was filled with electrolyte and sealed with liquid silicone sealant. The liquid silicone was allowed to dry and harden creating a seal. The thin cell has an exposed surface area of $\sim 48.9 \mathrm{~mm}^{2}$ with a distance of $0.55-0.75 \mathrm{~mm}$ between the electrodes.

\section{CNT film preparation and characterization}

The spray-painted CNT slides were imaged with an Atomic Force Microscope (Dual-Scan AFM, Pacific Nanotechnology, USA) to determine the coverage (figure 1). In addition, a probe station was used to measure the sheet resistance $R_{\square}(\Omega / \square)$ of the CNT films, by analyzing the distance dependence of the twoterminal resistance $R$ as a function of probe separation $L$ on a semilog scale and performing a least-squares fit to

$$
R=\frac{R_{\square}}{\pi} \log \left(\frac{L}{d}\right),
$$

where $d$ is the probe tip diameter. The counter electrodes used in this study were all obtained from the same batch in order to ensure uniformity and their sheet resistance was $R_{\square} \approx 50 \mathrm{k} \Omega / \square$.

\section{Solar Power Generation measurements}

The assembled cells were connected to a load resistor $R_{L}$ that was varied from 0 to $10 \mathrm{M} \Omega$ through a current amplifier and the voltage $V$ across and current $I$ through it are measured as a function of $R_{L}$ (figure 2). The cells were pointed straight at the sun and were measured in Northridge, CA (visibility: 10 miles, Latitude $=34^{\circ} \mathrm{N}$ ) at solar noon from Dec 2010 through April 2011. The sun's altitude $\beta$ was between $32.5^{\circ}$ and $65.7^{\circ}$, yielding an air mass of $A M=1 / \sin \beta \approx 1.7$. The average solar flux was $770 \mathrm{~W} / \mathrm{m}^{2}$. To minimize the effect of variability in solar conditions and cell assembly, devices were fabricated with large variations in carbon nanotube concentrations to highlight its effect on cell performance.

GNTs were created in batch operations, providing the ability to test various parameters and the resistances of electrodes used in experiments.

\section{Acknowledgments}

We thank Konstantin Daskalov, Hiral Patel, Ashkan Foroughi, Daniel Frochtzwajg, Karo Karapetyan, and Michael Darling for experimental assistance. This work is dedicated to the memories of Michael Dickson and Andrew Wieting.

\section{Author Contributions}

Conceived and designed the experiments: CK YP HP. Performed the experiments: CK YP HP. Analyzed the data: CK YP HP. Contributed reagents/materials/analysis tools: CK YP HP. Wrote the paper: CK YP HP.

high efficiency dye-sensitized solar cell. Chemical Communications. pp 2635-2637.

5. Shi D, Pootrakulchote N, Li R, Guo J, Wang Y, et al. (2008) New efficiency records for stable Dye-Sensitized solar cells with Low-Volatility and ionic liquid electrolytes. The Journal of Physical Chemistry C 112: 17046-17050.

6. Yella A, Lee H, Tsao HN, Yi C, Chandiran AK, et al. (2011) PorphyrinSensitized solar cells with cobalt (II/III)Based redox electrolyte exceed 12 percent efficiency. Science 334: 629-634. 
7. Baxter JB, Aydil ES (2005) Nanowire-based dye-sensitized solar cells. Applied Physics Letters 86: 053114

8. Law M, Greene LE, Johnson JC, Saykally R, Yang P (2005) Nanowire dyesensitized solar cells. Nature Materials 4: 455-459.

9. Bach U, Lupo D, Comte P, Moser JE, Weissortel F, et al. (1998) Solid-state dyesensitized mesoporous TiO 2 solar cells with high photon-to-electron conversion efficiencies. Nature 395: 583-585.

10. Saito Y, Kitamura T, Wada Y, Yanagida S (2002) Poly (3, 4-ethylenedioxythiophene) as a hole conductor in solid state dye sensitized solar cells. Synthetic Metals 131: 185-187.

11. Horiuchi T, Miura H, Sumioka K, Uchida S (2004) High efficiency of DyeSensitized solar cells based on Metal-Free indoline dyes. Journal of the American Chemical Society 126: 12218-12219.

12. Nazeeruddin MK, Kay A, Rodicio I, Humphry-Baker R, Mueller E, et al. (1993) Conversion of light to electricity by cis-X2bis(2,2'-bipyridyl-4,4' dicarboxylate)ruthenium(II) charge-transfer sensitizers ( $\mathrm{X}=\mathrm{cl}-$, br-, i-, CN-, and $\mathrm{SCN}-)$ on nanocrystalline titanium dioxide electrodes. Journal of the American Chemical Society 115: 6382-6390.

13. Kohle O, Ruile S, Gratzel M (1996) Ruthenium(II) Charge-Transfer sensitizers containing 4,4-Dicarboxy-2,2-bipyridine. synthesis, properties, and bonding mode of coordinated thio- and selenocyanates. Inorganic Chemistry 35: 4779-4787.

14. Nazeeruddin MK, Pchy P, Graetzel M (1997) Efficient panchromatic sensitization of nanocrystalline $\mathrm{TiO} 2$ films by a black dye based on a trithiocyanatoruthenium complex. Chemical Communications 1997: 1705-1706.

15. Aiga F, Tada T (2003) Molecular and electronic structures of black dye; an efficient sensitizing dye for nanocrystalline $\mathrm{TiO} 2$ solar cells. Journal of Molecular Structure 658: 25-32.

16. Boschloo G, Lindstrom H, Magnusson E, Holmberg A, Hagfeldt A (2002) Optimization of dye-sensitized solar cells prepared by compression method. Journal of Photochemistry \& Photobiology, A: Chemistry 148: 11-15.

17. Wang ZS, Yamaguchi T, Sugihara H, Arakawa H (2005) Significant efficiency improvement of the black dye-sensitized solar cell through protonation of TiO2 films. Langmuir 21: 4272-4276.

18. Vogel R, Hoyer P, Weller H (1994) Quantum-Sized PbS, CdS, Ag2S, Sb2S3, and Bi2S3 particles as sensitizers for various nanoporous Wide-Bandgap semiconductors. The Journal of Physical Chemistry 98: 3183-3188.

19. Li C, Mitra S (2007) Processing of fullerene-single wall carbon nanotube complex for bulk hetero-junction photovoltaic cells. Applied Physics Letters 91: 253112

20. Li C, Chen Y, Wang Y, Iqbal Z, Chhowalla M, et al. (2007) A fullerenesingle wall carbon nanotube complex for polymer bulk heterojunction photovoltaic cells. Journal of Materials Chemistry 17: 24062411.

21. Iijima S, Ichihashi T (1993) Single-shell carbon nanotubes of 1-nm diameter. Nature 363: 603-605.

22. Bethune DS, Klang CH, de Vries MS, Gorman G, Savoy R, et al. (1993) Cobalt-catalysed growth of carbon nanotubes with single-atomic-layer walls. Nature 363: 605-607.
23. Koo B, Lee D, Kim H, Lee W, Song J, et al. (2006) Seasoning effect of dyesensitized solar cells with different counter electrodes. Journal of Electroceramics 17: 79-82.

24. Trancik JE, Barton SC, Hone J (2008) Transparent and catalytic carbon nanotube films. Nano Letters 8: 982-987.

25. Ramasamy E, Lee WJ, Lee DY, Song JS (2008) Spray coated multi-wall carbon nanotube counter electrode for tri-iodide reduction in dye-sensitized solar cells. Electrochemistry Communications 10: 1087-1089.

26. Hwang S, Moon J, Lee S, Kim D, Lee D, et al. (2007) Carbon nanotubes as counter electrode for dye-sensitised solar cells. Electronics Letters 43: 1455-1456.

27. Kang M, Han Y, Choi H, Jeon M (2010) Two-step heat treatment of carbon nanotube based paste as counter electrode of dye-sensitised solar cells. Electronics Letters 46: 1509-1510.

28. Hu L, Hecht DS, Gruner G (2004) Percolation in transparent and conducting carbon nanotube networks. Nano Letters 4: 2513-2517.

29. Zhou Y, Hu L, Gruner G (2006) A method of printing carbon nanotube thin films. Applied Physics Letters 88: 123109.

30. Tans S, Verschueren A, Dekker G (1998) Room-temperature transistor based on a single carbon nanotube. Nature 393: 49-52.

31. Postma HWC, Teepen T, Yao Z, Grifoni M, Dekker C (2001) Carbon nanotube Single-Electron transistors at room temperature. Science 293: 76-79.

32. Yao Z, Postma HWC, Balents L, Dekker C (1999) Carbon nanotube intramolecular junctions. Nature 402: 273-276.

33. Postma HWC, de Jonge M, Yao Z, Dekker C (2000) Electrical transport through carbon nanotube junctions created by mechanical manipulation. Physical Review B 62: R10653-R10656.

34. Martel R, Schmidt T, Shea HR, Hertel T, Avouris P (1998) Single- and multiwall carbon nanotube field-effect transistors. Applied Physics Letters 73: 2447.

35. Odom TW, Huang J, Kim P, Lieber CM (1998) Atomic structure and electronic properties of single-walled carbon nanotubes. Nature 391: 62-64.

36. Wildoer JWG, Venema LC, Rinzler AG, Smalley RE, Dekker C (1998) Electronic structure of atomically resolved carbon nanotubes. Nature 391: 59-62.

37. Tang Y, Lee C, Xu J, Liu Z, Chen Z, et al. (2010) Incorporation of graphenes in nanostructured $\mathrm{TiO} 2$ films via molecular grafting for Dye-Sensitized solar cell application. ACS Nano 4: 3482-3488.

38. Mickelson ET, Huffman CB, Rinzler AG, Smalley RE, Hauge RH, et al. (1998) Fluorination of single-wall carbon nanotubes. Chemical physics letters 296: 188-194.

39. Rinzler AG, Liu J, Dai H, Nikolaev P, Huffman CB, et al. (1998) Large-scale purification of single- wall carbon nanotubes: process, product, and characterization. Appl Phys A 67: 29-37.

40. Vigolo B, Penicaud A, Coulon C, Sauder C, Pailler R, et al. (2000) Macroscopic fibers and ribbons of oriented carbon nanotubes. Science 290: 1331-1334.

41. Han L, Koide N, Chiba Y, Islam A, Mitate T (2006) Modeling of an equivalent circuit for dye-sensitized solar cells: improvement of efficiency of dye-sensitized solar cells by reducing internal resistance. Comptes Rendus Chimie 9: 645-651. 